\title{
NETWORKING OF ASTRONOMY INSTITUTIONS IN THE THIRD WORLD COUNTRIES
}

\author{
JAYANT V. NARLIKAR \\ Inter-University Centre for Astronomy and Astrophysics \\ Pune - 411007 , India. \\ e-mail: jvn@iucaa.ernet.in
}

\section{Introduction}

There are several international organizations currently working towards raising the standard of science and technology in the third world countries (TWCs). It is being appreciated that instead of dealing with the issue on a country by country basis, it helps to view and solve the problem in a network mode. It is sometimes the case that one TWC may have resource persons, or facilities which can benefit by sharing with the other TWCs. Or, a group of workers from different TWCs can be brought together for a school or workshop in which experts drawn from different parts of the world participate as lecturers or guides.

To address such problems a database is necessary to provide information on the scientific institutions in the Third World, so that member institutions of the database can have exchanges of information. The Third World Academy of Sciences (TWAS) has made a beginning by having the TWNSO - the Third World Network of Scientific Organizations.

What can be achieved by such a network? How can it be set up? In what way can the IAU and other international agencies help support it? I will deal with these issues next.

\section{What can the Network achieve?}

I can envisage the following activities for the Third World Astronomy Network (TWAN).

\subsection{VISITOR PROGRAMME}

There may be a few institutions in the TWCs who have experts at the international level in astronomy. Visits to these institutions by young scientists from other TWCs will help in training them in their respective fields. TWAS in fact welcomes and supports such 'south-south' visits.

\subsection{TWAN SCHOOLS AND WORKSHOPS}

A few institutions may be identified as nodal centres for holding introductory schools and advanced workshops for students from TWCs. The former may be along the lines of ISYAs of the IAU. The latter may be more specialized to frontier research areas and may invite a few experts from the developed countries. Funds for such meetings may be obtained from some international organizations like the TWAS, UNESCO, IAU, etc. as well as from the national agencies of the country where the meeting is held. This activity will take off if one such meeting is held per year in each of the nodal centres, there being, say $3-4$ of such centres.

\subsection{TWAN DATA CENTRES}

To facilitate quick transfer of data and allow easy access to journals, the TWAN may envisage setting up mirror sites for journals and data centres with the latest astronomical surveys, catalogues, etc. We may envisage one such facility each in Asia, Africa and Latin America. 


\subsection{USAGE OF OBSERVING FACILITIES}

A few TWCs may have good working telescopes, supported by instrumentation programmes. Astronomers from the TWCs may train for observing programmes and making instruments, at these places.

\section{Action points}

To achieve the above network the following steps could be taken :

(a) Prepare a directory listing all participating institutions (PIs) of the network. The directory should also list the strengths and interests of each PI. This will enable the deployment of personnel and facilities for the TWAN activities.

(b) Constitute an Executive Committee to formulate the academic programmes of TWAN and to allocate funds for them. The EC will, of course, have to acquire funds for these programmes.

(c) Identify a few nodal institutions to serve as TWAN centres for schools/workshops.

(d) Create databases/mirror sites for use by astronomers from the TWCs.

I hope that the IAU will take the initiative through its Working Group on World-Wide Development of Astronomy and its Commission 46 for Teaching of Astronomy. This may be a more focussed and specialized part of ICSU's activities for the TWCs. There is possibility of sponsorship/collaboration of TWAS.

Whatever the nature of support, I am confident that a TWAN-type organization can offer a lot for the nucleation and growth of astronomy in the TWCs.

\section{SUMMARY OF DISCUSSION DURING AFTERNOON SESSIONS (Percy to Hidayat)}

In discussion of Percy's paper, concern was expressed that, in becoming politically astute, astronomers might compromise their integrity. Percy did not feel that this need happen and reiterated that businessmen who are amateur astronomers can often be valuable allies. It was pointed out that simple observations that can be made without instruments often have great teaching value. For example, teaching people to see Haidinger's Plume (i.e. sky polarization) which is also useful for orienting one's self in strange territory and gives insight into animal and insect behaviour. Or the orientation of the Moon, at certain phases, with respect to the horizon illustrates the need for spherical coordinates.

Gerbaldi's,recommendation of the CLEA texts published by Cahiers Clairaut was strongly endorsed.

Nutridinov was congratulated for including history of astronomy in the bachelor's curriculum. It was pointed out that the International Centre for Theoretical Physics in Trieste has a programme of distributing books and journals to developing countries. Darrell Hoff has a similar programme.

Torres-Peimbert was urged to increase the number of students in the graduate programmes, even if first-generation students had difficulty finding jobs in astronomy. In Japan, some time ago, job opportunities in astronomy had been few and many graduating students went into business. Some professors tried to limit enrolment in graduate courses, but the numbers increased anyway.

After Fierro's paper, attention was drawn to the "Worldbeat" articles in the ASP magazine Mercury (including one by Fierro herself) and also to the ASP's quarterly teachers' newsletter.

Discussion on Crawford's paper centered on "astroeconomics", the relative cost-effectiveness of several small telescopes compared with one big one. Such comparisons work for bright objects, but, for faint ones, detector noise becomes relatively more important. Crawford quoted typical costs of $\$ 90,000$ (U.S.) for a good quality $0.5-\mathrm{m}$ telescope and $\$ 500,000$ (U.S.) for a $1-\mathrm{m}$.

Abt was asked if scientists should stop supporting overpriced commercial journals. He replied that many librarians are now boycotting them and astronomers should realize that papers in these journals are not now widely read.

Narlikar's appeal for IAU fostering of Third-World networks was endorsed. Perhaps the IAU could replicate at the international level networks that were already functioning well nationally. Caution was expressed. Again, working through ICSU seemed to be the way to go. Its programme of Capacity Building for Science was doing many of the things Narlikar described and, by collaborating, we would be strengthening all of science. 